\title{
LA TRADUCCIÓN ENTRE HEIDEGGER Y DERRIDA
}

\author{
PABLO BERNARDO SÁNCHEZ GÓMEZ
}

(UNED)

RESUMEN: La cuestión de la traducción, como afirma Derrida, es «la» cuestión de la deconstrucción. En este sentido, comprende Derrida que la traducción no consiste en la «transferencia» de significantes entre las lenguas, proceso que respetaría los significados ideales-inmateriales. Por el contrario, Derrida apunta hacia una traducción de un "original» que nunca ha sido, un texto que necesita esencialmente de la traducción y, por tanto, afirma la traducción como una tarea «im-posible», como la continua creación del otro y de lo otro, como recepción de la herencia e imposible afirmación del duelo. En este sentido, cabría plantear la posibilidad de «traducir» los textos de Derrida hacia el pensamiento heideggeriano, donde también se establecería de manera explícita el rechazo a la noción de traducción en cuanto «intercambio», es decir, como tarea técnica o mecánica, apuntando así hacia la traducción como la verdadera esencia del pensamiento y del lenguaje. No obstante, se comprueba en esta aproximación la im-posibilidad de traducir los textos de Derrida al corpus heideggeriano.

PALABRAS CLAVE: aporía; deconstrucción; Derrida; imposibilidad; promesa; traducción.

\section{Translation between Heidegger and Derrida}

ABSTRACT: The question about translation, as Derrida claims, is «the» question of deconstruction. Thus, Derrida understands that translation is not just the "transfer» of signifiers between languages, process which would respect the ideal-immaterial meaning. On the contrary, Derrida points to a translation of an «original» which has never been, a text which needs translation and so he states translation as an «im-possible» task, as the endless creation of the Other, as reception of the legacy and the impossible affirmation of mourning. In this sense, it may be set out the possibility of "translating" Derrida's texts towards Heideggerian thought, where it is also rejected the idea of a translation as «exchange», as a technical or mechanical task, stating then translation as the truly essence of thought and language. However, it is found out on this closeness the im-possibility of translating Derrida's text to Heideggerian corpus.

KEY WORDS: aporia; deconstruction; Derrida; impossibility; promise; translation.

Uno de los momentos más explícitos en los que Heidegger plantea la capitalidad de la cuestión en torno a la traducción es en su curso del año 1942 dedicado al himno de Hölderlin «El Ister». Allí afirma que «la dificultad de una traducción no es nunca un mero asunto técnico, sino que concierne a la relación del hombre con la esencia del mundo y con la dignidad del lenguaje. Dime lo que piensas de la traducción y te diré quién eres» ${ }^{1}$. En esta misma dirección que pretende destacar o subrayar la importancia del preguntar de forma temáticamente explícita por la tarea de la traducción señala Derrida que «la cuestión de la deconstrucción es de un extremo al

1 Heidegger, M., Hölderlins Hymne "Der Ister», Vittorio Klostermann, Frankfurt am Main, 1984, p. 76. 
otro la cuestión de la traducción y de la lengua de los conceptos, del corpus conceptual de la metafísica llamada “occidental” »². Siendo así que ambos autores posicionan la experiencia de la traducción como uno de los elementos determinantes de la historia de «la» filosofía "occidental», cabría preguntarse si esta afinidad entre Heidegger y Derrida apuntaría, en cierta medida, hacia un «querer decir» (vouloir-dire) «lo mismo» (Selbe) que posibilitaría una traducción entre ambos pensamientos, esto es, una relación biunívoca que haría de sus textos una expresión de lo idéntico (Identisch). En este sentido, la cuestión sería doble. En primer lugar, habría que señalar si tanto Heidegger como Derrida traducen un mismo y único pensamiento para, posteriormente, determinar si los textos derridianos no consistirían más que en paráfrasis, comentarios y traducciones elaboradas sobre la obra de Heidegger.

Si esto fuese posible debería, para comenzar, delimitarse un concepto de traducción homogéneo y estabilizado en el interior tanto de la obra de Heidegger como en los textos de Derrida. Sin embargo, de este último, como señala Ángeles Carreres, y aun cuando son numerosas las ocasiones en las que Derrida considera expresamente la cuestión de la traducción, no resulta posible obtener o sintetizar una unidad conceptual. Por el contrario, el término «traducción» se comporta en Derrida como una constelación de hasta ocho direcciones de lectura (la traducción como transformación, como traslado, como transposición, como interpretación, como reformulación, como intercomunicación, como traducción interlingual y como transcripción), encontrándose por tanto la propia noción de «traducción» sujeta al proceso mismo de traducción, de desplazamiento ${ }^{3}$. En este sentido, siendo el término "traducción» un haz de direcciones irreconciliables en un núcleo semántico, éste podría constituir un término «indecidible», de los cuales afirma Derrida que resultan, necesaria e inevitablemente, intraducibles ${ }^{4}$, entendiendo aquí por «traducción» la transferencia de uno o varios significados hacia un mismo sentido estable y nuclear.

En lo que respecta a Heidegger, éste habría señalado ya en el fragmento anteriormente citado que la traducción no consiste, o no «meramente», en la sustitución o intercambio de un significante por un supuesto equivalente en otra lengua, como si de un simple proceso mecánico o automático se tratase. De ser así, la traducción se comprendería desde el respeto a la pureza e integridad del significado a traducir, el cual permanecería en todo momento del proceso de traducción idéntico a sí mismo, siendo los significantes, encarnaciones contingentes en una lengua concreta del significado incorpóreo, los únicos elementos que se verían alterados. La traducción «técnica», por tanto, correspondería a lo que Jakobson denomina

2 Derrida, J., «Lettre à un ami japonais» en Psyché. Inventions de l'autre, Galilée, Paris, 1987 , p. 387.

3 Cfr. Carreres, A., Cruzando limites. La retórica de la traducción en Jacques Derrida, Peter Lang, Bern, 2005, pp. 60-69.

4 Afirma Derrida en «Qu'est-ce qu'une traduction 'relevante'?» que «la mayor parte de las palabras llamadas «indecidibles» que me han interesado desde hace tiempo son también, y esto no tiene nada de fortuito, intraducibles en una sola palabra (pharmakon, suplemento, différance, himen, etc.) y su lista no puede, por definición, cerrarse. DERrIDA, J., «Qu'est-ce qu'une traduction "relevante”?», en Marie-Louise Mallet; Ginette Michaud (ed.), L'Herne. Derrida. Éditiones de l'Herne, Paris, 2004. p. 575. 
«traducción interlingual» $\mathrm{O}$ «traducción propiamente dicha » ${ }^{5}$, proceso al que Geoffrey Be-nnington identifica con «Google Translate» y afirma como la más distante posible de la noción heideggeriana de la tarea de traducción ${ }^{6}$. Porque esta comprensión de la traducción es, para Heidegger, dependiente en su totalidad de la noción aristotélica de signo, la cual se habría erguido como fundamento último de toda reflexión metafísica en torno a la esencia del lenguaje. Así, en la lengua de la metafísica, «las letras son signos de los sonidos, éstos de los padecimientos del alma y éstos son, a su vez, signos de las cosas (Zeichen der Dinge). La vertebración de la estructura está configurada por la relación sígnica» ${ }^{7}$. Siendo el lenguaje comprendido como representación de los significados, la traducción «técnica» en Heidegger se vería supeditada a la función expresivo-representativa, esto es, a un «querer-decir». De este modo, toda traducción se encontraría ya inserta en el ámbito o epocalidad de la verdad en cuanto adecuación, «ónoí $\omega \sigma ı \varsigma$ », siendo por tanto esta comprensión «técnica» de la traducción un efecto necesario de la transformación de la esencia de la verdad comenzada por Platón y consumada en Aristóteles ${ }^{8}$, es decir, una necesidad interna del pensamiento metafísico.

Por su parte, Derrida señala ya desde sus primeros textos cómo el intento de determinar la dimensión lingüística como algo suplementario, accesorio e irrelevante, siendo así dependiente del supuesto significado ideal-inmaterial, se ha desarrollado, en un sentido u otro, a través de «la» historia de la metafísica ${ }^{9}$. En este sentido, y poniendo en directa relación la constitución del signo lingüístico con cierto proceso de traducción, cita Derrida en «Le puits et la pyramide» a Hegel, quien afirma que «el signo es una cierta intuición inmediata que representa un contenido totalmente diferente del que tiene para sí misma — la pirámide [subraya Hegel] en la que un alma extraña (eine fremde Seele) es transportada [traspuesta, trasplantada, traducida: verzetzt; versetzsen es también empeñar; im Leihause versetzen: dejar en el Monte de Piedad] y guardada (aufbewahrt: confiada, consignada,

5 Cfr. Jakobson, R., Language in Literature, Harvard University Press, Massachusetts, 1987, p. 429.

6 Cfr. Bennington, G., «Heidegger and Google». Conferencia impartida en la European Graduate School, Saas Fee, 16 de Agosto de 2016, cuya grabación se encuentra disponible: «http://www.youtube.com/watch?v=uf-IA5_ru6s»

7 Heidegger, M., Unterwegs zur Sprache, Vittorio Klostermann, Frankfurt am Main, 1985,p. 192 (traducción española: De camino al habla, Ediciones del Serbal-Guitard, Barcelona, 1987, p. 182).

8 Cfr. Heidegger, M., Wegmarken, Vittorio Klostermann, Frankfurt am Main, 1976, pp. 231-233 (traducción española: Hitos, Alianza Editorial, Madrid, 2000, pp. 192-194).

9 De sobra conocidos son los momentos en los que Derrida, en sus primeros textos, señala esta «metafísica de la presencia» que pretendería establecer un núcleo de pura idealidad de significado incorrupta por la contingencia del significante. Cfr. La Voix et le Phénomène, PUF, Paris, 1967, pp. 58-59 (traducción española: La Voz y el Fenómeno, PreTextos, Valencia, 1985, pp. 101-102). Cfr. De la grammatologie. Les éditions de minuit, Paris, 1967,. pp. 46-47 (traducción española: De la gramatología, Siglo XXI, México, 1986, pp. 40-42. Cfr. La dissémination, Éditions du Seuil, Paris, 1972, pp. 104-105 (traducción española: La diseminación, Editorial Fundamentos, Madrid, 1997, pp. 137-139). 
puesta en consigna)» ${ }^{10}$, algo que Derrida retoma en Glas $^{11}$. De este modo, Derrida coincide con Heidegger en comprender la «traducción» desarrollada metafísicamente como dependiente de la estructura sígnico-representativa del lenguaje y, por tanto, supeditada al valor de "presencia», de «intuición» pura y originaria representada o encarnada como significado en el significante.

Si el signo es la reunión del significante o la indicación (Zeichen) con el «quererdecir» (Bedeutung) de la auténtica realidad interno-ideal del alma, la traducción no consistiría más que en la sustitución de una serie determinada de diferentes significantes referidos todos ellos a un mismo contenido, a un mismo significado. Así, para asegurar el valor de presencia, la tarea de «la» filosofía habría consistido en la determinación clara y distinta de las relaciones entre los significantes y sus significados asociados, delimitando así el «corpus» lingüístico que permitiría, puesto que el pensamiento tiene que vérselas con el uso de signos, de representantes, desarrollar el deseo arque-tele(te)ológico de la disolución del lenguaje mismo en aras de la idealidad incorrupta del significado. La «cosa» de la filosofía son los significados y, por tanto, los significantes no deben impedir su correcto ejercicio, o al menos lo mínimo posible, llegando incluso hasta la «insignificancia». Para ello, los referentes de los significantes deben ser perfectamente conocidos y determinados, evitando cualquier posible dificultad de comprensión que pudiese dificultar la transferencia de una lengua a otra sin pérdida esencial para el pensamiento: «la tesis de la filosofía es la tesis de la traducibilidad» ${ }^{12}$. De este modo, una vez delimitadas las

10 Derrida, J., Marges de la philosophie, . Les éditions de minuit, Paris, 1972, p 96 (traducción española: Márgenes de la filosofía, Cátedra, Madrid, 1994, p. 119). En este mismo sentido debe leerse el siguiente fragmento: «Avant de savoir comment et que traduire par "représentation", on doit s'interroger sur le concept de traduction et de langage, qui est souvent dominé par le concept de représentation, qu'il s'agisse de traduction interlinguistique, intralinguistique (à l'intérieur d'une seule langue) ou même, pour recourir ici par commodité à la tripartition de Jakobson, de traduction intersémiotique (entre des langages discursifs et des langages non discursifs), dans l'art par exemple. Chaque fois nous retrouvons la présupposition ou le désir d'une identité de sens invariable, déjà présente derrière tous les usages et réglant toutes les variations». Derrida, J. «Envoi», en Psyché. Inventions de l'autre, ed. cit., p. 116.

11 Cfr. Derrida, J., Glas, Galilée, Paris, 1974, 7b; 152a.

12 Cito en extenso debido a su capitalidad para nuestra cuestión: «el programa del paso a la filosofía significa en este contexto, me parece, que la operación filosófica, si es que está dotada de alguna originalidad y especificidad, viene definida como un proyecto de traducción, como fijación de un cierto concepto de traducción y como proyecto de traducción. ¿Qué dice la filosofía? Si podemos fabular sobre ¿qué es la filosofía? ¿Qué dice un filósofo cuando es filósofo? Dice: lo que importa es la verdad o es el sentido, y el sentido es previo a la lengua o se encuentra más allá de la lengua y es, por consiguiente, intraducible. Es el sentido lo que ordena y, en consecuencia, debe poder fijarse la univocidad del sentido o en todo caso la plurivocidad debe ser controlable, y si esta plurivocidad es controlable, la traducción, como transporte de un contenido semántico a otra forma significante, a otra lengua, es posible. No hay filosofía a menos que sea posible la traducción, por lo que la tesis de la filosofía es la traducibilidad, la traducibilidad en el sentido corriente, el transporte de un sentido, de un valor de verdad, de una lengua a otra, sin daño esencial». DeRRIDA, J., L'oreille de l'autre. Otobiographies, transferts, traductions, VLB Éditeur, Montréal, 1982,. p. 159 (traducción española: La oreja del otro. Traducción y autobiografía, Editorial Carpe Noctem, Madrid, 2016, pp. 134-135). 
relaciones significantes-significados, bastaría con consultar la correspondencia entre significantes en un diccionario bilingüe para realizar una traducción «adecuada». Respecto a este modo de traducción Heidegger se manifiesta expresamente en contra, afirmando que "una palabra no es ningún instrumento ${ }^{13}$, algo que sirviese para otra cosa (representación del significado ajeno a ella) y, por tanto, el lenguaje no puede comprenderse, no por lo menos exclusivamente, como la transmisión o intercambio económico de significados ideales envueltos en significantes lingüísticos contingentes. De nuevo en el curso de año 1942, «El Ister», afirma Heidegger:

Ciertamente, desde que comprendemos el lenguaje (Sprache) como un mero vehículo (Verkehrsmittel), entonces el diccionario, ligado a la técnica del tránsito (Verkehr) e intercambio (Austausches), está «al mando» (Ordnung). Visto de cara al espíritu histórico de un lenguaje (geschichtlichen Geist einer Sprache), por el contrario, ningún diccionario aporta inmediatamente la medida. Esto es cierto para toda traducción (Übersetzung), porque toda traducción debe necesariamente cumplir la transición desde el espíritu de una lengua (Sprachgeist) a la de otra. No hay traducción si nos referimos a que una palabra de una lengua podría, o incluso debería, sustituir como equivalente de otra palabra en otra lengua ${ }^{14}$.

La traducción en cuanto «trasvase» o «transporte», la traducción «interlingual» supone necesariamente para Derrida que «existe una lengua, que existe una traducción en el sentido propio, es decir, un paso de una lengua a otra; y si no está asegurada la unidad del sistema lingüístico, toda esta conceptualización en torno a la traducción se pone en peligro» ${ }^{15}$. De no ser así, de no poder hermetizarse e higienizarse el cuerpo de la lengua, no podría constituirse un núcleo puro e incontaminado de la misma que hiciese a la lengua esa lengua que es y, por tanto, no sería posible conformar el diccionario que sustentaría la traducción como transferencia ${ }^{16}$. De nuevo, Derrida ya había apuntado en De la grammatologie y La Voix et le Phénomène hacia esta "contaminación originaria»: la (a)lógica de la huella obliga a comprender la constitución de lo propio como la remisión a lo otro y, por tanto, la identidad se constituye desde la alteridad ${ }^{17}$, pudiendo así Derrida enunciar que «el afuera es el adentro» ${ }^{18}$, siendo así necesario comprender la irrupción de lo

13 Heidegger, M., Parmenides, Vittorio Klostermann, Frankfurt am Main, 1982, p. 21 (traducción española: Parménides, Ediciones Akal, Madrid, 2005, p. 22).

14 Heidegger, M., Hölderlins Hymne «Der Ister». ed. cit., p. 75. Otro ejemplo: «es cierto que los diccionarios traducen, correctamente, res adversae por desgracia y res secundae por suerte; pero sobre aquello que las palabras dicen, como lo dicho pensado, los diccionarios dicen poco. En realidad, aquí, y en los otros casos, no es que nuestro pensamiento viva de la etimología, sino que la etimología queda remitida a considerar primero las relaciones esenciales de aquello que las palabras, como elementos que forman sintagmas nombran de un modo no desplegado». Heidegger, M., Vorträge und Aufsätze, Vittorio Klostermann, Frankfurt am Main, 2000, p. 175 (traducción española: Conferencias y artículos, Ediciones del Serbal, Barcelona, 1994, p. 151)

15 Derrida, J., L'oreille de l'autre. ed. cit., p. 134 (traducción española, p. 113).

16 Cfr. Derrida, J., Glas, ed. cit., 14a.

17 Cfr. Derrida, J., La Voix et le Phénomène,. ed. cit. pp. 75-76 (traducción española, p. 121). Cfr. Derrida, J., De la grammatologie, ed. cit., pp. 68-69 (traducción española, p. 61).

18 DerRida, J., op. cit., p. 65 (traducción española, p. 57). 
otro como anterior a la constitución del «ipse» ${ }^{19}$. No obstante, tratando de forma temática la cuestión de la traducción, Derrida recurre a la lectura del relato bíblico sobre la Torre de Babel para señalar esta imposibilidad de auto-posición identitaria. En «Des tours de Babel» ${ }^{20}$ afirma Derrida que esta torre «no señala solamente la multiplicidad irreductible de las lenguas, ella exhibe un inacabamiento, la imposibilidad de completar, de totalizar, de saturar, de acabar cualquier cosa que fuese del orden de la edificación, de la construcción arquitectural, del sistema y de la arquitectónica» ${ }^{21}$.

El relato narra la historia de los Shem, cuyo nombre significa en hebreo, precisamente, «nombre». Este pueblo pretendía construir una torre que ascendiese hasta el cielo, hasta Dios, y de este modo ser capaces de darse a sí mismos su nombre. Para ello, la lengua de los Shem debía ser única, imponerse violentamente a toda la realidad, imponiendo su «labio» y consumando así el deseo íntimo de la traducción, es decir, hacer a la lengua irrelevante e innecesaria: la lengua de los Shem sería el perfecto «oírse-hablar» sin pérdida, el permanecer inalterado e incontaminado por cualquier elemento extraño a ellos mismos, afirmando de este modo la cima o punta de su torre, su «cap», sin necesidad de recurrir a ningún otro ${ }^{22}$. No obstante, en el momento en el que los Shem se encuentran ascendiendo en la construcción de su torre, Dios interrumpe la obra clamando su propio nombre, dando su nombre, «Babel», que puede ser comprendido en hebreo, a causa de su homofonía, como "confusión» ${ }^{23}$. De este modo, como señala Derrida, Dios impone y al mismo tiempo prohíbe la traducción: en cuanto nombre propio, nombre de Dios, «Babel» resulta intraducible pero, al mismo tiempo, si el pueblo de los Shem puede comprender «Babel» como "confusión», la traducción ya se ha realizado: es la aporía de la singularidad y de su legibilidad, de la unicidad y su repetición ${ }^{24}$. Así, lo que vendría a señalar este relato es que desde el momento en el que hay nombre,

19 Cfr. Derrida, J., Sur Parole. Instantanés philosophiques, Éditions de l'Aube, Paris, 1999, p, 66 (traducción española: ¡Palabra! Instantáneas filosóficas, Editorial Trotta, Madrid, 2001, p. 51).

20 El título resulta ya, por sí mismo, intraducible. Por una parte, indica hacia «las torres de Babel», señalando así la necesidad de concebir la necesidad de la multiplicación de las lenguas. Al mismo tiempo, puede el título referir, por homofonía, a «détour», desvío, es decir, a la imposibilidad de erguir «la» torre de Babel, a la necesidad de la "différance».

21 Derrida, J., «Des tours de Babel», en Psyché. Inventons de l'autre, ed. cit., p. 203. Por otra parte, en torno a la cuestión de la arquitectura, Cfr. DerRidA, J., Les arts de l'espace, Éditions de la Différence, Paris, 2015, p. 58.

22 Derrida, J., L'autre cap. Les éditions de Minuit, Paris, 1991. p. 31 (traducción española: El otro cabo. Ediciones del Serbal, Barcelona, 1992, p. 29)

${ }_{23}$ Como señala Derrida, «Él [Dios] les impone la confusión, al mismo tiempo que impone su nombre propio, el nombre que ha elegido y que quiere decir confusión y que los Shems entienden en su lengua, confusamente, por confusión». DerRIDA, J., L'oreille de l'autre, ed. cit., p. 136 (traducción española, p. 115).

${ }_{24}$ Esta aporía entre el hecho singular, irrepetible y único, propio, y la generalidad que permite su lectura y por tanto su repetición, iterabilidad y traducción es tratada extensamente en la reflexión que Derrida desarrolla en torno a la «fecha» en Schibboleth. Cfr. DERRIDA, J., Schibboleth pour Paul Celan, Galilée, Paris, 1986. p. 22-23 (traducción española: Schibboleth. Para Paul Celan,. Editora Nacional, Madrid, 2002, p. 17) 
desde el momento en el que Dios da (su) nombre, éste no puede aquietarse en sí mismo, no puede realizarse en una identidad, sino que se da a la traducción, se da a la alteridad y a la iterabilidad. El nombre propio, la firma, en principio intraducible por tener un solo y único referente singular e irrepetible se entrega, sin embargo, a la repetición, perdiéndose a sí misma entre las traducciones ${ }^{25}$.

En este sentido, la cuestión de la traducción ya no se articularía desde el binomio "posible-imposible», es decir, bajo la pregunta "¿es posible y bajo qué circunstancias es posible la traducción?», sino desde la aporética relación «imposible-necesario», desde la «im-posibilidad», desde la posibilidad de lo imposible y por tanto desde la infinita responsabilidad de la traducción. Es esta experiencia aporética de la traducción la que Derrida lee en Ulysse gramophone a través de la oración, presente en Finnegans Wake, "And he war». Estas tres palabras, enunciadas en una obra en la que se expresan más de cuarenta lenguas ${ }^{26}$, plantean la indecibilidad del nombre de Dios tal y como sucedía en el relato babélico: por una parte, el término alemán «war», pasado del verbo «sein» que recogería la resonancia bíblica del «Yo soy»; por otra, el «war» inglés, que recoge la traducción francesa del Finnegans Wake como «il guerre», "él guerra». Dios es guerra, pero siéndolo abre su haber sido en y hacia otra lengua. Dios es guerra habiendo sido en y como la multiplicación de las lenguas, es decir, como traducción, como confusión ${ }^{27}$. Y así, si no hay traducción «adecuada» para los «indecidibles», como habíamos señalado anteriormente, la oración "and he war» abre la necesidad de la decisión en la traducción, la responsabilidad absoluta hacia el acontecimiento que es la im-posibilidad de la traducción misma ${ }^{28}$.

25 «¿Hay firmas? Sí, por supuesto, todos los días. Los efectos de firma son la cosa más corriente del mundo. Pero la condición de posibilidad de estos efectos es simultáneamente, una vez más, la condición de su imposibilidad, de la imposibilidad de su pureza rigurosa. Para funcionar, es decir, para ser legible, una firma debe poseer una forma repetible, iterable, imitable; debe poder desprenderse de la intención presente y singular de su producción. Es su mismidad lo que, alterando su identidad y su singularidad, divide el sello». DerRIDA, J. «Signature, événement, contexte», en Marges de la philosophie. ed. cit. p. 391-392 (traducción española, p. 371).

26 Cfr. Derrida, J., Ulysse gramophone, Galilée, Paris, 1987, p. 16.

27 Cfr. Derrida, J., op. cit., pp. 38-39.

28 «¿Qué sería un camino sin aporía? ¿Lo habría sin aquello que lo abre donde no está abierto, ya esté cortado o aún sepultado en el no-camino? No puedo pensar un camino sin la necesidad de decidir cuando la decisión parece imposible. Ni una decisión, y por lo tanto una responsabilidad, cuando la decisión ya es posible y programable». Derrida, J., Sauf le nom, Galilée, Paris, 1993, p. 109 (traducción española: Salvo el nombre, Amorrortu Editores, Buenos Aires, 2011, p. 88). Sobre la im-posibilidad del acontecimiento, afirma Derrida: "cuando lo imposible se hace posible, el acontecimiento tiene lugar (posibilidad de lo imposible). Ésta es precisamente, irrefutablemente, la forma paradójica del acontecimiento: si un acontecimiento es solamente posible, en el sentido clásico de la palabra, si se inscribe en unas condiciones de posibilidad, si no hace más que explicitar, desvelar, revelar, realizar lo que ya era posible, entonces ya no es un acontecimiento. Para que un acontecimiento tenga lugar, para que sea posible, es preciso que sea, como acontecimiento, como invención la venida de lo imposible [...] Toda responsabilidad debe pasar por esa aporía que, lejos de paralizarla, pone en movimiento un nuevo pensamiento de lo posible. Le garantiza su ritmo y su respiración: diástole, sístole y síncope, latido de lo im-posible, de lo imposible como 
La traducción es, por tanto, inevitable e imposible. Ahora bien, para habitar en esta aporía sin convertirla en una mera paradoja resulta necesario ampliar la noción «clásica» de la traducción, esto es, la traducción comprendida como "paso» de una lengua a otra. En este sentido, afirma Heidegger llevando a cabo lo que podría comprenderse como una «traducción intralingual» que la «traducción» (Übersetzen) consiste, efectivamente, en un «transporte» (Übertragung), en una «transposición», pero siempre y cuando esta última sea comprendida en cuanto «transducción» (Übersetzen $)^{29}$. De este modo, para Heidegger la «traducción» (Übersetzen) como «transducción» (Übersetzen) no consistiría en la mera reproducción de una palabra en otra lengua diferente, es decir, en la «imitación» o «reproducción» de lo otro en lo propio de mi lengua. Así, la corrección de una traducción, sostiene Heidegger, no puede consistir en la «exactitud» o «corrección» literal, en la erudición y adecuación filológico-lingüística, sino en la fidelidad al «asunto» o «cosa» propia de la palabra a traducir ${ }^{30}$, siendo así posible para Heidegger afirmar que «una traducción puede ser prácticamente correcta, pero de acuerdo con su asunto, es decir, en cuanto interpretación, ser completamente falsa ${ }^{31}$, no diciendo, por consiguiente, nada $^{32}$. De este modo, la transducción (Übersetzen) es siempre un salto ${ }^{33}$, un adentrarse en lo todavía por pensar en la palabra a traducir, una interpretación esencial y, por tanto, una traducción que piensa en la esencia del lenguaje en cuanto abis$\mathrm{mo}^{34}$. En este mismo sentido que apunta hacia una traducción que no «imitaría» tornándose invisible frente al «original» sino que lo transforma afirma Derrida:

Es en efecto, en el horizonte de una traductibilidad absolutamente pura, transparente y unívoca, que se ha constituido el tema de un significado trascendental. En los límites en donde ella es posible, al menos donde parece posible, la traducción practica la diferencia entre significado y significante. Pero si esta diferencia no es pura nunca, la traducción tampoco lo es, y a la noción de traducción habrá que suplantarla por la noción de transformación: transformación reglamentada de

condición de lo posible». Derrida, J., Papel Máquina, Editorial Trotta, Madrid, 2003,. pp. 257258. Por otra parte, señala Derrida: «La "deconstrucción” ha sido definida a menudo como la experiencia misma de la posibilidad (imposible) de lo imposible, de lo más imposible, condición que comparte con el don, con el "sí", el "ven", la decisión, el testimonio, el secreto, etc.». DerRidA, J., Sauf le nom. ed. cit. p. 32 (traducción española, p. 27)

29 En la traducción española del curso Parmenides realizada por Carlos Másmela «Übersetzen» se traduce por «Transportar», «Übersetzen» por «traducir» y «Übertragung» por «transposición». Aquí se opta, sin embargo, por seguir la traducción propuesta por Raúl Gabás en ¿Qué significa pensar? de los términos «Übersetzen» por «transducción», «Übersetzen» por «traducción», mientras que «Übertragung» se traducirá por «transporte».

30 Cfr. Heidegger, Holzwege, Vittorio Klostermann, Frankfurt am Main, 1977, p. 322 (traducción española: Caminos de bosque, Alianza Editorial, Madrid, 1995, p. 240)

31 Heidegger, M., Wegmarken. ed. cit., p. 244 (traducción española, p. 203)

32 Cfr. Heidegger, M., Grunbegriffe, Vittorio Klostermann, Frankfurt am Main, 1991, p. 109. (traducción española: Conceptos fundamentales, Alianza Editorial, Madrid, 1999, p. 156)

33 «La transducción (Übersetzen) sólo se logra en un salto». HeidegGer, M., Wass heisst Denken?, Vittorio Klostermann, Frankfurt am Main, 2002, p. 236 (traducción española: ¿Qué significa pensar?, Editorial Trotta, Madrid, 2005, p. 190).

${ }_{34}$ «La traducción pensante (denkende Übersetzen) hacia aquello que en la sentencia llega al lenguaje, es el salto por encima del abismo (Sprung über einen Graben)». HeIDEgGer, M., Holzwege, ed. cit., p. 329 (traducción española, p. 245) 
una lengua por otra, de un texto por otro. Nosotros no tendremos ni hemos tenido que ver nunca con cierto «transporte» de significados puros, que el instrumento —o el «vehículo»— significante dejaría virgen e intocado, de una lengua a otra, o al interior de una lengua misma ${ }^{35}$.

Parecería, por tanto, que tanto Heidegger como Derrida comprenden la tarea de la traducción de forma equivalente, tomando ambos distancia de la traducción en cuanto «transporte» y apuntando hacia la noción de «transformación» o de transducción (Übersetzen). Sin embargo, en esta aparente coincidencia se torna evidente la infinita y discreta distancia que tensa entre Heidegger y Derrida, precisamente en el modo mismo en el que el primero plantea, de forma temática, la cuestión de la traducción. De este modo, cuando Heidegger plantea sus críticas a la noción de traducción en cuanto «transferencia» lo hace siempre en el interior de une reflexión en torno a los términos que considera "claves», "fundamentales» (Grundwort) o «conductores» (Leitwort) del pensamiento. Así, habría para Heidegger textos que podrían traducirse de forma técnica, mecánicamente, casi automáticamente; textos tales como cartas o informes que no presentarían resistencia alguna al proceso de «Übersetzen» corriente y que no plantearían mayor problema. Por el contrario, Heidegger se mostraría más interesado en esos textos de los que se experiencia su esencia en el proceso mismo de traducción, es decir, en cuanto esencialmente intraducibles, entre los cuales destaca, como texto «eminente», el poema (Dichtung) ${ }^{36}$. De este modo, cuando Heidegger plantea la cuestión de la traducción como "problema», de lo que se trataría es, como señala Zarader, de pensar «las experiencias (Erfahrungen) iniciales, experiencias posibles por la lengua del inicio (en este caso, la lengua griega), y que fueron depuestas, al mismo tiempo que conservadas, en un cierto número de palabras fundamentales (Grundworte)» ${ }^{37}$, esto es, esas palabras que conservaría el eco del primer destello de la verdad y de la esencia del ser. De este modo, podría parecer adecuada la sugerencia de Derrida de que "hay siempre en Heidegger, dependiente de otras cuestiones, el deseo nostálgico de reencontrar el verdadero nombre, el único nombre del Ser» ${ }^{38} y$, por tanto, cierto remanente de una noción de la traducción en cuanto transferencia, en cuanto adecuación a un sentido.

No obstante, Heidegger insiste en señalar que la traducción no conduce una palabra extranjera (en este caso, términos griegos) hacia la lengua receptora (el alemán), sino más bien la segunda hacia la primera, a lo en ella dicho en cuanto no-dicho, es decir, a lo impensado incluso para quienes usaron el término en

35 Derrida, J., Positions, Les Éditions de Minuit, Paris, 1972, p. 31 (traducción española: Posiciones, Pre-Textos, Valencia, 1977, P. 55).

36 "No da igual decir "traducir" (Übersetzen) cuando se trata de una carta de negocios que decir "traducir" cuando se trata de un poema (Gedicht). La carta es susceptible de traducción, el poema no». Heidegger, M., Der Satz vom Grund, Vittorio Klostermann, Frankfurt am Main, 1997, p. 145 (traducción española: La proposición del fundamento, Ediciones del Serbal, Barcelona, 2003, p. 138).

37 Zarader, M., Heidegger et les paroles de l'origine, Librairie Philosophique J. VRIN. Paris, 1986, 22.

38 DerridA, J., «La déconstruction et l'autre» en: Les Temps Modernes. n. 669-670, juliooctubre 2012, pp. 10-11. 
primer lugar ${ }^{39}$. Por tanto, para Heidegger, la traducción de las «Grundworte» no puede tratarse, en modo alguno, de adecuación a un sentido dado. Así, si el pensar es para Heidegger el oír las señas que nos hace la palabra ${ }^{40}$, el «original» necesita siempre la transducción (Übersetzen), la interpretación ${ }^{41}$, no siendo ésta por tanto algo sobrevenido, un añadido que no afectaría en lo esencial al «significado»: no hay significado último si por tal se entiende un objeto o un sentido disponible. De este modo, si la traducción de las «Grundworte» es para Heidegger el encontrarse en camino hacia lo por-pensar en esa palabra misma, en el encaminarse hacia la esenciarse del ser, la traducción dirige al pensamiento hacia la dimensión o ámbito originario de esa palabra y en el que esa palabra (se) da como indicación ${ }^{42}$. Las palabras fundamentales no recogen significados, sino ecos ${ }^{43}$, el resonar insonoro de «la» palabra (das Wort) o sentencia (Sprache) ${ }^{44}$ en su darse. En este sentido, lo que podría denominarse la «traducción fundamental» es, para Heidegger, la aproximación ilimitada hacia aquello que sólo en el proceso de traducción mismo muestra su esencia en cuanto inasible llamada hacia sí, es decir, en cuanto convocación hacia lo por-pensar: traducir es encaminarse hacia aquello que en la palabra hace guiños (winken $)^{45}$.

La traducción es, de este modo, promesa hacia aquello a lo que se dirige, encontrándose así orientada hacia el porvenir del «original» y no hacia un mero texto pasado y por tanto disponible a la mano. De este modo, podría comprenderse a Javier Marías cuando afirma que «la traducción es una cuestión de la memoria» ${ }^{46}$, añadiendo que esta memoria, sin embargo, se debería entender en cuanto "Andenken» o «Gedächtnis», pero no en como «Erinnerung»: la traducción señala el pasado como porvenir, la memoria como porvenir. Siendo en este sentido en el que Heidegger comprende la traducción de las «Grundworte», Derrida afirma, tras apuntar la posible añoranza heideggeriana de un pretérito para siempre pasado, que «para ser justo, sin embargo, se pueden encontrar numerosos pasajes donde

39 Cfr. Heidegger, M., Wegmarken, ed. cit., p. 244 (traducción española, p. 203).

40 «Tenemos que oír la palabra tomada literalmente, de tal forma que atendamos a las indicaciones que dan cuenta de la palabra. En dicho atender tomamos entonces en consideración lo que la palabra trata de decir. Nosotros ejercitamos la atención. Comenzamos a pensar». Heidegger. M., Parmenides, ed. cit., p. 22 (traducción española, p. 24).

${ }_{41}$ En su curso sobre "El Ister», señala Heidegger que «todo traducir debe ser una interpretación (Auslegung). Y lo mismo a la inversa: toda interpretación es traducir». HeIDEgGer, M., Hölderlins Hymne «Der Ister», ed. cit., p. 75.

42 Cfr. Heidegger, M., Was heisst Denken?, ed. cit., p. 178 (traducción española, p, 148).

43 Cfr. Heidegger, M., Unterwegs zur Sprache, ed. cit., p. 218 (traducción española, 207).

44 «En la consideración de lo "literal" (Wörtlichen) nos parece que se toma en serio la palabra. No obstante, desatendemos las palabras mientras sólo tengamos interés en las "palabras". La traducción "literal" ("wörtliche" Übersetzung) no puede reproducir simplemente las palabras y "enriquecer" de este modo el lenguaje de traducción con palabras "nuevas", inusuales y con frecuencia desproporcionadas, sino que tiene que ir más allá de las palabras y considerar las palabras mismas. La erudición acerca de las palabras no garantiza aún un saber de las palabras. Éstas dicen lo propiamente decible: la palabra, la sentencia (das Wort, das Spruch)». Heidegger, M., Parmenides, ed. cit., p. 21 (traducción española, p. 24).

45 Cfr. Heidegger, M., Unterwegs zur Sprache, ed. cit., p. 109 (traducción española, 104).

46 Marías, J., Literatura y fantasma, Siruela, Madrid, 1994, p. 110. 
Heidegger es frontalmente crítico consigo mismo y renuncia a su nostalgia ${ }^{47}$. Si el lenguaje es el espacio del darse como porvenir en cuanto traducción, el supuesto «original», el texto «base», el texto «fuente» necesita, desde su «origen», la traducción. De este modo, si el original sólo deviene tal como promesa en la traducción, el texto a traducir, el origen, no es ni un mero pasado, algo simplemente muerto y perdido en la traducción, ni algo vivo, algo perfectamente presente que convertiría a la traducción un mero añadido: el original sobre-vive en la traducción. Esto es algo que Derrida encuentra en el texto de Walter Benjamin «La tarea del traductor», donde éste señala:

Así como las manifestaciones de la vida están íntimamente relacionadas con todo ser vivo, aunque no representan nada para éste, también la traducción brota del original, pero no tanto de su vida como de su «sobrevida» (Überleben), pues la traducción (Übersetzung) es posterior al original. Y sin embargo, para las obras importantes que nunca encuentran a sus traductores adecuados en la época de su creación, indica la fase de su supervivencia (Fortleben). La idea de la vida y de la supervivencia (Fortleben) de las obras debe entenderse con un rigor totalmente exento de metáforas ${ }^{48}$.

Así como Derrida planteaba en un primer momento la aporía de la traducción como la experiencia de lo imposible y al mismo tiempo necesario, de la singularidad y de lo legible-repetible, ahora señala su imposibilidad como estado de sobre-vida, ni vida ni muerte y, por tanto, ni «original» ni «copia», ni pureza ni suplemento $^{49}$. En este sentido, como la herencia misma, la traducción supone el cortocircuito entre lo constatativo, la recepción de algo dado, y lo performativo, la creación por parte del receptor de eso dado ${ }^{50}$, siendo así, por tanto, algo que nunca llega a cerrarse, un texto del que el heredero, receptor o traductor nunca puede

47 Derrida, J., «La déconstruction de l'autre», ed. cit. p. 11. En otro texto afirma Derrida : «no se trata en cualquier caso de volver a la lengua griega, y menos aún de presuponer un olvido absoluto disimulado en la lengua griega, una lengua archi-materna, una abuelita de la lengua griega que sería absolutamente virgen e intocable [...] Se trata de una operación del pensamiento por la que debemos traducirnos, dice él [Heidegger], en algún lugar, en el pensamiento olvidado de otra lengua; tenemos que traducirnos a ese pensamiento, no hacer venir eso a nuestra lengua, sino, al contrario, ir hacia el pensamiento impensado de la otra lengua». J. DeRrida. L'oreille de l'autre, ed. cit., p. 152 (traducción española, p. 129).. La cuestión de la tensión entre nostalgia y promesa en Heidegger es tratada en numerosas ocasiones por Derrida. Véase, por ejemplo, Derrida, J., Mémoires pour Paul de Man, Galilée, Paris, 1988, p. 135-142 (traducción española: Memorias para Paul de Man, Gedisa, Barcelona, 2008, pp. 142-149).

48 Benjamin, W., Angelus Novus, Edhasa, Barcelona, 1971, p. 129.

49 Señala Derrida en Parages «un texto no vive más que si sobre-vive, y sólo sobrevive si es a la vez traducible e intraducible (siempre a la vez, $y$ : ama, al "mismo tiempo"). Totalmente traducible, él desaparece como texto, como escritura, como cuerpo de lengua. Totalmente intraducible, incluso en el interior de lo que se cree que ser una lengua, muere inmediatamente. La traducción triunfante no es por tanto ni la vida ni la muerte del texto, únicamente o ya su supervivencia». Derrida, J., Parages, Galilée, Paris, 1986, pp. 147-148.

50 Cfr. DerRIDA, «Psyché. Invention de l'autre» en Psyché. Inventions de l'autre, ed. cit., p. 35. 
llegar a «reapropiarse ${ }^{51}$. Esta misma (a)lógica se expone y realiza en Derrida a través de diferentes lecturas, siendo quizá aquí la más interesante aquella que refiere a la «imposible afirmación del duelo». Frente al duelo «melancólico», frente a la actividad de la «Erinnerung» que hace del otro "pasado» algo pretérito y, por tanto, algo aquietado, homogéneo, resto ontologizado en una tumba ${ }^{52}$ susceptible de ser apropiado inmediatamente en la digestión ${ }^{53}$, susceptible de ser $\operatorname{archivado}^{54}$, Derrida plantea la imposibilidad de clausurar el recuerdo, es decir, de darle un forma "apropiada», "adecuada» a aquello que se está recordando: aquello que (se) nos da (desde) el otro, el "original», se mantiene abierto, en continua llamada como convocación de y hacia el otro que en cuanto tal otro, en cuanto radicalmente otro, no puede ser nunca apropiado. Así, Derrida plantea de nuevo la necesidad de experienciar la aporía, de «evitar a toda costa la buena conciencia» ${ }^{55}$ y asumir el fracaso del duelo, el fracaso de la traducción, precisamente como su mayor "éxito»" ${ }^{56}$. En este sentido, la traducción es el duelo ${ }^{57}$ por el «original», es decir, el «llevar permanentemente el luto por lo que nunca se tuvo ${ }^{58} \mathrm{y}$, por tanto, una tarea aporética sin fin.

En esta misma línea, las numerosas ocasiones que Heidegger dedica a reflexionar en torno a la pérdida de la esencia de la verdad del ser en la traducción histórica del pensamiento griego a la lengua latina ${ }^{59}$ no tendría, en modo alguno, una intención melancólica. Porque lo que le interesa a Heidegger de las «Grundworte» griegas no es el hecho de que pertenezcan a un supuesto pasado glorioso del pensamiento, momento inaugural de la claridad, sino su capacidad de nombrar (Nennen), es decir, su habilidad o capacidad de «llevar-a-mostrarse» (zum-sich-zeigen-bringen ${ }^{60}$ la

51 «La herencia es aquello de que no puedo apropiarme, lo que me corresponde y cuya responsabilidad tengo, que recayó en mí en el reparto pero sobre lo cual no tengo derecho absoluto. Heredo algo que también debo transmitir: ya sea esto chocante o no, no hay derecho de propiedad sobre la herencia. Ésa es la paradoja. Siempre soy el locatario de una herencia. Su depositario, su testigo o su relevo... No puedo apropiarme de ninguna herencia en su totalidad. Comenzando por la lengua». Derrida, J., Ecografías de la televisión, Eudeba, Buenos Aires, 1998, p. 138.

52 Cfr. Derrida, J., Spectres de Marx, Galilée, Paris, 1993, p. 30 (traducción española: Espectros de Marx, Editorial Trotta, Madrid, 1998, p. 23).

53 Cfr. Derrida, J., Glas, ed. cit., 40b.

54 Cfr. Derrida, J., Mal d'archive, Galilée, Paris, 1995, p. 56 (traducción española: Mal de archivo. Editorial Trotta, Madrid, 1997, 41.

55 Derrida, J., Apories. Mourir - s'attendre aux «limites de la vérité», Galilée, Paris, 1996, p. 42 (traducción española: Aporías. Morir - esperarse (en) «los límites de la verdad», Paidós, Barcelona, 1998, p. 40).

${ }_{56}$ Cfr. Derrida, J., Mémoires pour Paul de Man, ed. cit., p. 54 (traducción española, p. 45).

57 Cfr. Derrida, J., "Qu'est-ce qu'une traduction “relevante"?», ed. cit., p. 574.

58 Derrida, J., Le monolinguisme de l'autre, Galilée, Paris, 1996, p. 61.

59 Cfr. Heidegger, M., Holzwege, ed. cit., pp. 7-9 (traducción española, pp. 16-17). Aquí se encuentra también un ejemplo de la capitalidad que concede Heidegger a la tarea de la traducción: «¿qué una mera traducción ha podido provocar todo esto? Pues bien, tal vez esto nos enseñe a considerar lo que puede llegar a pasar en una traducción». HeIDEGGER, M., op. cit., p. 371 (traducción española, p. 276).

60 Cfr. Heidegger, M., Einführung in die Metaphysik, Vittorio Klostermann, Frankfurt am Main, 1983, p. 179 (traducción española: Introducción a la metafísica, Gedisa, Barcelona, 2001, p. 155). 
verdad del ser en cuanto esenciarse-ocultante. De este modo, gracias a su natural "parentesco ${ }^{61}{ }^{1}$, la tarea del pensamiento consistiría en aproximar la lengua alemana hacia aquello im-pensado en las palabras iniciales griegas, hacia aquello nunca dicho y, sin embargo, contenido como indicación o guiño en ellas. En este sentido, la traducción alemana de la lengua griega debe alcanzar a pensar «más griegamente» que los griegos mismos ${ }^{62}$, es decir, más originariamente que el «original». La traducción, por tanto, nos lleva más allá o más acá del original, trasladándonos y nombrando el espacio o la dimensión inaugural del ser ${ }^{63}$. De este modo, Heidegger comprende que esta «transducción» (Übersetzen) abriría el espacio para el «otro inicio» (andere Anfang) desde el "primer inicio» (erste Anfang), es decir, traduciría el «original» re-traduciendo las traducciones latinas que habrían olvidado el destello inicial contenido en las palabras fundamentales griegas y logrando, en este proceso de «Übersetzen», destacar la necesidad del olvido mismo, la necesidad de esas traducciones ocultadoras. La transducción que propone Heidegger del «primer inicio» conduce, por tanto, en la dirección de «otro inicio» más «originario» que el primero, siendo así la transducción no simplemente reproducción sino verdadera inauguración ${ }^{64}$. La pérdida y olvido del guiño, la ocultación de la esencia «original» del ser en las sucesivas traducciones acontecidas históricamente no supone para Heidegger, por tanto, un accidente o una contingencia que podría haberse evitado

${ }_{61}$ Sobre esta hermandad entre el griego y el alemán, no habría más que consultar los seminarios dedicados a Hölderlin, especialmente aquellos pasajes en los que Heidegger se detiene en las traducciones de los poetas griegos realizadas por Hölderlin (Cfr. HeIDEGger, M., Hölderlins Hymne «Der Ister», ed. cit., pp. 153-180). Aquí citamos, sin embargo, la famosa frivolidad que Heidegger afirma en su entrevista en el Spiegel: "pienso en el particular e íntimo parentesco de la lengua alemana con la lengua de los griegos y con su pensamiento. Esto me lo confirman hoy una y otra vez los franceses. Cuando empiezan a pensar, hablan alemán; aseguran que no se las arreglan con su lengua». HeIDEgGer, M., Entrevista del Spiegel, Tecnos, Madrid, 1996, pp. 91-92.

62 «Nuestro pensamiento tiene por tarea pensar lo pensado por los griegos de modo aún más griego». HeIdegger, M., Unterwegs zur Sprache, ed. cit., p. 127 (traducción española, p. 122).

63 "La "traducción" (Übersetzen) no es en absoluto un mero traslado de las palabras griegas a la fuerza de nuestra propia lengua. No pretende sustituir la palabra griega, sino únicamente y precisamente trasladarnos hasta ella y en cuanto tal traslado desaparece en ella». Heidegger, M., Wegmarken, ed. cit., p. 245 (traducción española, p. 204).

64 «El otro inicio del pensar (der andere Anfang des Denkens) es llamado así, no por ser sólo de otra forma que cualquier otra filosofía vigente, sino porque tiene que ser el único otro a partir de la referencia al único y primer inicio (einen und ersten Anfang). Desde esta asignación recíproca del uno y otro inicio está también ya determinado el tipo de meditación pensante (denkerischen Besinnung) en tránsito (...) En el saber del pensar transitorio queda el primer inicio decididamente como primero y sin embargo ha sido superado (überwunden) como inicio. Para este pensar tiene que ir el más claro respeto por el primer inicio, que abre así su singularidad, junto con la desconsideración del apartamiento de otro preguntar y decir (anderen Fragens und Sagens)». Heidegger, M., Beiträge zur Philosophie (Vom Ereignis), Vittorio Klostermann, Frankfurt am Main, 1989, p. 5-6 (traducción española: Aportaciones a la filosofía. Acerca del evento, Editorial Biblos, Buenos Aires, 2003, p. 23. Traducción modificada). 
de haber prestado una mayor atención durante el proceso de traducción ${ }^{65}$. El texto «original», esencialmente, se oculta en la traducción y, al mismo tiempo, se aproxima en cuanto esa misma ocultación, resultando así la traducción la explicitación de su necesario y esencial recusación (Verweigerung). En este mismo sentido que afirma la deuda contraída originariamente con la traducción por parte del «original» señala Derrida:

El original no es algo pleno que de forma casual recibe una traducción. La situación del original es la situación de solicitud, es decir, de una falta, de un exilio: el original está a priori en deuda con la traducción. Su supervivencia es una exigencia de traducción, un deseo de traducción, de una manera parecida a la exigencia de Babel: «tradúceme» ${ }^{66}$.

El imperativo o la orden dada por el texto «original», esta necesidad de la traducción, no puede sino relacionarse con aquella otra, presentada ya con respecto a la herencia, que Derrida denomina «espectralidad». El espectro no tiene nada propio, no dispone de una forma o de una figura, de un «cuerpo», observándonos por tanto sin poder ser contemplado en su propiedad, característica ésta que Derrida denomina "efecto visera ${ }^{67}$. Así, el espectro se nos dirige, se nos presenta como retorno de algo que no ha tenido "primera vez», encomendándosenos así con su orden: «léeme» ${ }^{68}$. De este modo el espectro, como el «original», no es «algo» que pueda traducirse «adecuadamente», sino la ruina originaria (il y a la ruine) ${ }^{69}$, la ceniza que resta ahí (il y a là cendre), que produce efectos de «restancia» sin poder nunca resucitar como ave Fénix, es decir, sin poder remontar hasta aquello que hubo antes del holocausto ${ }^{70}$. En este sentido, como afirma Derrida, la traducción tiene vocación de ruina ${ }^{71}$, se enfrenta con algo que no «es» y que sin embargo se da: es esta la ley del texto ${ }^{72}$.

Junto a la ceniza, la ruina y el espectro Derrida plantea la cuestión de la traducción desde la noción de «secreto». Así, afirma, la traducción lo sería de un secreto (il y a du secret ${ }^{73}$ ), pero no de un secreto en cuanto ocultación que pudiese ser desvelado, esto es, el secreto comprendido como resguardo de una esencia, sino

65 Cfr. Heidegger, M., Bessinung, Vittorio Klostermann, Frankfurt am Mai, 1997, pp. 195-196 (traducción española: Meditación, Editorial Biblos, Buenos Aires, 2006, p. 171). Cfr. Heidegger. M., Parmenides, ed. cit., pp. 72-75 (traducción española, pp. 65-68).

${ }_{66}$ Derrida, J., L'oreille de l'autre, ed. cit., p. 201 (traducción española, p. 172).,

67 Cfr. Derrida, J., Spectres de Marx, ed. cit., p. 26 (traducción española, p. 21).

68 DERridA, J., op. cit., p. 40 (traducción española, p. 30).

69 Cfr. Derrida, J., Mémoires d'aveugle, Éditions de la Réunion des Musées Nationaux, Paris, 1990, p. 72.

70 Cfr. Derrida, J., La difunta ceniza, La Cebra Ediciones, Buenos Aires, 2009, pp. 25-29.

71 «la traduction, au sens strict, traditionnel et dominant de ce terme, rencontre une limite insurmontable —et le commencement de sa fin, la figure de sa ruine (mais peut-être une traduction est-elle vouée à la ruine, à cette forme de mémoire ou de commémoration qu'on appelle une ruine ; la ruine est peut-être sa vocation mais aussi un destin qu'elle accepte dès l'origine». DerridA, J., «Qu'est-ce qu'une traduction “relevante”?», ed. cit. p., 565.

72 «El texto se guarda, como la ley. No habla sino de sí mismo, y por eso también de su no-identidad consigo. Ni llega ni deja llegar a sí. Es la ley, hace la ley y deja al lector ante la ley». Derrida, J., Prejuzgados. Ante la ley, Avarigani Editores, Madrid, 2011, p. 61.

73 Derrida, J., Dar la muerte, Paidós, Barcelona, 2000, p. 113. 
de un secreto que no por desvelarse resulta menos secreto, un secreto más allá del esquema «ocultación-desocultación», «esotérico-exotérico» ${ }^{74}$. De este modo, la traducción lo es del secreto que no oculta nada sino que consiste en la pura relación en la que algo, el espectro, se nos dirige y nos obliga a responder, en la que algo nos llama y nos reclama ${ }^{75}$. No hay, en modo alguno, «núcleo» del secreto que deba ser desvelado en la traducción, sino que es precisamente la falta, la necesidad de la falta, lo que mueve y pone en obra la traducción, la respuesta ${ }^{76}$. Derrida plantea, por tanto, la insuficiencia de considerar la traducción como mero vehículo de un texto a otro, pues «nunca se trata del mismo texto $»^{77}$ al no haber tal cosa como «el» original inafectado por el proceso de traducción. El «original», en definitiva, «se da modificándose, su don no es un objeto dado, vive y sobrevive en mutación ${ }^{78}$, siendo así siempre la traducción un proceso «relevante» de y para el «original $»^{79}$.

Es en este mismo sentido en el que debería leerse a Heidegger cuando afirma que «traducimos correctamente si decimos lo mismo. Ahora bien, ¿qué significa lo mismo? ¿Equivale a lo "idéntico"? De ninguna manera» ${ }^{80}$. Heidegger señala de este modo, en una suerte de confidencia personal, que a lo largo de las múltiples traducciones que ha realizado ha sentido la inconmensurabilidad que distancia las lenguas, la esencial diferencia consigo del lenguaje y, sin embargo, siempre ha intuido o presagiado que las lenguas compartían un mismo origen, que «la esencia

74 Cfr. Derrida, J. Passions, Galilée, Paris, 1993, p. 60.

75 Cfr. Derrida, J., Dar la muerte, ed. cit., p. 143.

76 «La Ananké es que no hay núcleo intacto, nunca lo ha habido, y es eso lo que se quiere olvidar, el olvido ue queremos olvidar de algún modo. No es que algo haya sido olvidado. Lo que queremos olvidar es que no hay nada que olvidar, que nunca ha habido un núcleo intacto y que eso, ese fantasma, ese deseo de núcleo intacto es lo que pone en marcha cualquier deseo, lengua, llamada, interpelación». DerRIDA, J., L'oreille de l'autre, ed. cit., p., 152 (traducción española, p. 130).

77 DERRIDA, J., op. cit., p. 208 (traducción española, p. 179).

78 Derrida, J., «Des tours de Babel» en Pysché. Inventions de l'autre, ed. cit., p. 217.

79 Intraduciblemente, Derrida plantea la traducción como «relevance», lo cual puede tener tres sentidos en español: «elevación», «relevación» (Derrida traduce aquí «Aufhebung», como en otros textos, por «relève») y «sazonar», "condimentar», aportar un nuevo sabor (assaisonner). Cfr. DERRIDA, J., "Qu'est-ce qu'une traduction "relevante”?», ed. cit., pp. 572-573.

80 Heidegger, M., Was heisst Denken?, ed. cit., p. 244 (traducción española, p. 198). La diferencia entre «lo mismo» y «lo igual» es recurrente en Heidegger, aunque quizá donde la exponga con mayor claridad sea en "...Poéticamente habita el hombre...», donde afirma: «Lo mismo (das Selbe) no coincide nunca con lo igual (dem Gleichen), tampoco con la vacía indiferencia de lo meramente idéntico (Identischen). Lo igual se está trasladando continuamente a lo indiferenciado, para que allí concuerde todo. En cambio lo mismo es la copertenencia de lo diferente desde la coligación (Versammlung) que tiene lugar por la diferencia (Unterschied). Lo mismo sólo se deja decir cuando se piensa la diferencia. En el portar a término decisivo de lo diferenciado adviene a la luz la esencia coligante de lo mismo (im Austrag des Unterschiedenen kommt das versammelnde Wesen des selben zum Leuchten). Lo mismo aleja todo afán de limitarse sólo a equilibrar lo diferente en lo igual. Lo mismo coliga lo diferente en una unión originaria. Lo igual, en cambio, dispersa en la insulsa unidad de lo que es uno sólo por ser uniforme». Heidegger, M., Vorträge und Aufsätze, ed. cit., p. 196197 (traducción española, p. 168). 
de dos lenguas radicalmente distintas era la misma» ${ }^{81}$, que las lenguas convergían en la misma cima ${ }^{82}$. La tarea de la traducción, por tanto, señala hacia la esencia del lenguaje, hacia ese lugar (Ort) que es la punta de lanza y que revela el darse del lenguaje en cuanto esenciarse que guiña. Como señala Ireland, Heidegger «entiende la traducción como la única oportunidad para el desocultamiento inicial del lenguaje (das Wesen der Sprache). Y esta oportunidad se presenta en el modo en el cual la traducción responde a la extrañeza y lo absolutamente extranjero, que reclama una profunda traducción en el desocultamiento del lenguaje» ${ }^{83}$. De este modo, esta cima se correspondería con lo que Heidegger denomina «Aufriss» y «Sage», el «Trazo Abriente» y el «Decir», que roturarían e inscribirían los modos del decir, los trazos (Riss), siendo de este modo las «Leitworte» caminos que conducirían, sin alcanzarla nunca, hacia «la» palabra (das Wort), no pudiendo por tanto llegar a traducirse plenamente ${ }^{84}$. Nuestras palabras escuchan (hören) «la» palabra (das Wort) y en este sentido le pertenecen (gehören) siempre, son respuestas que intentan llevar el habla, como habla, al habla (die Sprache als die Sprache zur Sprache bringen), es decir, traducir «la» palabra en palabras ${ }^{85}$. No obstante, en cuanto todo decir es ya un hablar, toda palabra pertenece al despliegue del Decir y, por lo tanto, nunca puede decirlo "propiamente», nunca abarca o remonta hasta su origen ${ }^{86}$.

Toda palabra es ya traducción de un envío, de un «decir confiador» (Zusage) que se da dividiéndose, sustrayéndose, multiplicando sus trazos, retraduciéndose. En este mismo sentido, señala Derrida en «Nombre de oui» que hay un «sí» anterior a cualquier enunciación, afirmativa o negativa y, por lo tanto, un «sí» que no puede ser abarcado ni desde las palabras ni desde un supuesto metalenguaje, sino al que sólo podemos aproximarnos prometiéndolo ${ }^{87}$ en una traducción interminable que nunca llega a cerrar el círculo de la reapropiación, de la traducción adecuada ${ }^{88}$ : toda palabra es, por tanto, traducción que da como resultado "palabras oblicuas»" La traducción promete su objeto, y por tanto la traducción es la confirmación, la

${ }^{81}$ Heidegger, M., Unterwegs zur Sprache, ed. cit., p. 109 (traducción española, p. 105).

82 Cfr. Heidegger, M., Hölderlin Hymne «Der Ister», ed. cit. p., 76.

83 Ireland, J. A., «Heidegger, Hölderlin, and Eccentric Translation», en Schalow, F. (ed.). Heidegger, Translation and the Task of Thinking, Springer, London, 2011, p. 254.

84 «La palabra Ereignis, pensada a partir del asunto indicado, debe hablar ahora como palabra conductora (Leitwort) al servicio del pensar. Pensada como palabra conductora, se deja traducir (überstzen) tan poco como la palabra conductora griega $\lambda$ ó ${ }^{\prime} \varsigma$, o la china, Tao». HeIDEgger, M., Identidad y diferencia, Anthropos, Barcelona, 1990, p. 87.

85 Cfr. HeIdegger, M., Unterwegs zur Sprache, ed. cit., pp. 242-249 (traducción española, pp. 228-236).

${ }_{86}$ «No podemos abarcar el despliegue del habla (Sprachwesen) porque nosotros, que sólo podemos decir en cuanto que re-decimos el Decir (die Sage nachsagen), pertenecemos dentro del Decir». Heidegger, M., op. cit., p. 254 (traducción española, p, 240).

${ }_{87}$ Cfr. Derrida, J., «Nombre de oui» en Psyché. Inventions de l'autre, ed. cit., pp. 644-645.

88 Cfr. Derrida, J., Ulysse gramophone, ed. cit., pp.132-133.

${ }^{89}$ «Desde que hay palabra, y esto puede decirse de la huella en general y de la posibilidadoportunidad (chance) que ella es, la intuición directa, ella, no tiene ninguna oportunidadposibilidad (chance). Se puede denunciar la palabra "oblicua", pero no podemos negar la indirección destinerrante desde que hay la huella». Derrida, J., Passions, ed. cit., p. 68. 
actualización de la promesa misma, la puesta en marcha de la promesa. De este modo, siguiendo de nuevo a Walter Benjamin, señala Derrida:

Lo que las lenguas alcanzan intencionalmente cada una y en conjunto en la traducción, es la lengua misma como acontecimiento babélico, una lengua que no es la lengua universal en el sentido de Leibniz, una lengua que no es tampoco la lengua natural que cada una sigue siendo por su cuenta, es el ser-lengua de la lengua, la lengua o el lenguaje en cuanto tales, esta unidad sin ninguna identidad consigo misma que hace que haya las lenguas y que sean lenguas ${ }^{90}$.

El ser-lengua de la lengua en cuanto «différance» hace de la traducción la promesa im-posible de la reconciliación ${ }^{91}$. Así, si bien Derrida señala que «el origen de la filosofía es la traducción, la tesis de la traducibilidad, de tal modo que allí donde la traducción fracasa en este sentido, lo que fracasa es ni más ni menos que la filosofía ${ }^{92}$, Heidegger reivindica, no obstante, el nombre de «filosofía» para esa tarea que no consiste ya más en el intercambio mercantil de significados dados sino, por el contrario, en el "corresponder que trae a un lenguaje la llamada del ser»" La filosofía sería para Heidegger, por tanto, la interminable traducción del nombre del ser, del darse (d)el nombre. En este sentido, si Dios da (su) nombre, «Babel», toda palabra, como señala Derrida, portaría, conduciría hacia Dios. De este modo, afirma Derrida que las palabras nombran a Dios, dejan hablar a Dios en ellas, se encomiendan a Dios, pero precisamente así lo señalan como aquello que no puede ser nombrado, como aquello a lo que uno se dirige a través del nombre sin nombrarlo propiamente ${ }^{94}$ : "hace falta el nombre" querría decir que el nombre falta: debe faltar, hace falta un nombre que falte. Al lograr entonces borrarse, él mismo estará a salvo » ${ }^{95}$. De nuevo, Derrida señala así la aporía de la traducción imposible de «Babel»: el nombre de Dios se da en cada nombre, se borra en cada nombre y así se sostiene como promesa. De este modo, Dios sería el espacio de la traducción y de la promesa, el dar espacio a la traducción y a la promesa, pero también eso prometido y traducido. La traducción es, por tanto, la promesa infinita del nombre de Dios, promesa que sólo se cumple en su nunca poder llegar a realizarse ${ }^{96}$. Ahora bien, si la multiplicación de las lenguas, la multiplicación de los trazos son guiños o direcciones que conducen hacia Dios, la lengua materna de cada uno nunca pertenece,

90 DerridA, J., «Des tours de Babel», en Psyché. Inventions de l'autre, ed. cit., p. 232.

91 «La traducción hace presente de un modo solamente anticipador, anunciador, casi profético, una afinidad que no es jamás presente en esa presentación». Derrida, J., op. cit., p. 220.

92 Derrida, J., L'oreille de l'autre, ed. cit., pp. 159-160 (traducción española, p. 135).

93 Heidegger, M., "Was ist das - die Philosophie?» en Identität und Differenz, Vittorio Klostermann, Frankfurt am Main, 2006, p. 26. (traducción española: ¿Qué es la filosofía?, Herder, Barcelona, 2004, p. 66).

94 Cfr. Derrida, J., Sauf le nom, ed. cit., pp. 60-61 (traducción española, p. 50).

95 DERRIDA, J., op. cit., p. 80 (traducción española, p. 65).

96 «No se logra jamás una traducción en el sentido puro o absoluto del término; lo que logra una traducción es la promesa de un logro, de una reconciliación. De igual modo, hay traducciones que no logran esta promesa. Una buena traducción es una traducción que realiza este acto performativo que llamamos promesa, es decir, por medio de la traducción vemos anunciarse una posible reconciliación entre las lenguas». Derrida, J., L'oreille de l'autre, ed. cit., p. 163 (traducción española, pp. 138-139). 
nunca puede ser del todo propia, pues se encuentra ya siempre traduciendo(se) en el nombre de Dios: «Babel». La lengua, de este modo, es siempre la traducción de una pre-primera lengua que está por venir, traducción de una lengua que nunca ha tenido lugar, una lengua del espectro, de Dios o del cualquier/radicalmente otro ${ }^{97}$. De este modo, la lengua propia es siempre "plus d'une langue», una lengua del otro y de lo otro, una lengua ya siempre habitada por la traducción. Así, si la lengua es el hacer sitio a la pre-primera lengua por venir en «mi» lengua, venida que sólo llega sin terminar nunca de llegar en la traducción, ésta no puede consistir sino en la creación misma de la pre-primera lengua en cada una de las traducciones desarrolladas: cuanto más resistente y reticente sea la traducción, cuanto más imposible resulte, más espacio se proporciona a la venida del acontecimiento, a la creación im-posible, a la promesa de Dios, a la reconciliación. Es por esto por lo que Derrida señala que, en definitiva, lo que más le gustaría sería dedicarse a traducir el poema más absolutamente intraducible ${ }^{98}$. En este sentido, siguiendo a Heidegger ${ }^{99}$, afirma Derrida:

No hay lengua dada, o más bien, hay la lengua, hay donación de lengua (es gibt die Sprache), pero una lengua no es. Nada dado. Ella no existe. Llamada, ella llama, como la hospitalidad del anfitrión aun antes de toda invitación. Prescribiente, ella queda por ser dada, ella no permanece más que con esta condición: quedar aún por ser dada ${ }^{100}$.

Nos encontramos ya siempre en el espacio de la traducción, incluso en el interior de nuestra propia lengua ${ }^{101}$, si bien no en el sentido de la «traducción intralingual» de Jakobson, pues la lengua no traduce sus términos entre sí sino que se dirige a la absoluta heterogeneidad que la constituye originariamente. De este modo, simulando el título de uno de los textos de Derrida, podría quizá hablarse de «le retrait de la traduction»: al no pretender la traducción el sentido "propio» de su objeto, al no disponer de un objeto «último» en su tarea, la traducción, como la metáfora ${ }^{102}$, no puede más que re-trazarse, reelaborarse, reanudarse y prometer de nuevo, dar un giro de más que no llega a clausurar el círculo. Así, si como señala Derrida «la

97 Cfr. Le monolinguisme de l'autre, ed. cit., p. 118.

98 Derrida, J., Moscou aller-retour, Éditions de l'Aube, Paris, 1995, p. 118.

99 «Nunca podremos decir de la palabra: ella es, sino: ella da (es gibt), no en el sentido de que "se den" palabras, sino en cuanto sea la palabra misma la que da. La palabra: la donante (das Gebende)». HeIdegger, M., Unterwegs zur Sprache, ed. cit., p. 182 (traducción española, p. 173).

100 Derrida, J., Le monolinguisme de l'autre, ed. cit., pp. 125-126.

101 Esta traducción, señala Heidegger, es, pese a lo que podría parecer, la más compleja: «la tarea más difícil es siempre la traducción del propio lenguaje en su palabra más propia. Así, por ejemplo, la traducción de la palabra de un pensador alemán al lenguaje alemán es particularmente difícil, porque aquí domina el terco prejuicio de que nosotros, quienes hablamos alemán, entenderíamos la palabra alemana sin ninguna dificultad, por cuanto pertenece, después de todo, a nuestro propio lenguaje, mientras que, por el contrario, para traducir una palabra griega tenemos que aprender primero esa lengua extranjera». HeIDEGGER, M., Parmenides, ed. cit., p. 18 (traducción española, p. 20).

102 Derrida pone en directa conexión la metáfora con la traducción (Übertragung y Übersetzung). Cfr. DERRIDA, J., «Le retrait de la métaphore» en Psyché. Inventions de l'autre, ed. cit., p. 76. 
metáfora siempre ha sido definida como el tropo del parecido» ${ }^{103}$, al no consistir la traducción en la relación de parentesco o familiaridad entre las lenguas o consigo misma, sino en la afirmación de la diferencia que promete la reconciliación, se tendría que afirmar, tal y como hace Derrida con la metáfora, su «movimiento catastrófico» ${ }^{104}$, es decir, el paso a la catacresis ${ }^{105}$, a la generalización del movimiento de traducción y, por tanto, a su infinitud. Así, el objeto último de la traducción resulta, por tanto, intraducible y, sin embargo, no puede dejar de traducirse.

En este sentido, la cuestión que se planteaba al comienzo se comprende ahora como ingenua. Desde el momento en el que tanto Heidegger como Derrida toman distancia de una comprensión de la traducción como "transferencia» o "vehículo» del significado, apuntando ambos hacia la infinita tarea de la traducción imposible, no puede siquiera plantearse la intención de adecuar el pensamiento de uno a los textos del otro, esto es, señalar la identidad de su «querer-decir» que tomaría las diferencias como meras «contingencias materiales». El propio Derrida, siendo interrogado sobre su «familiaridad» con Heidegger, señala que «los textos de Heidegger están siempre delante de nosotros; su riqueza interpretativa es inagotable, lo que le hará ser leído y releído durante los siglos que vienen» ${ }^{106}$. Así, es también fácil comprender que Derrida afirme que «nada de lo que intento habría sido posible sin la apertura de las cuestiones heideggerianas» ${ }^{107} \mathrm{y}$ al mismo tiempo confiese no poder leer a Heidegger sino estrábicamente, balanceándose así entre la admiración y la ironía, entre la deuda y la crítica ${ }^{108}$. Porque Derrida traduce a Heidegger, lo asume como tarea, como fantasma que se le dirige entre las ruinas reclamando ser leído, exigiendo ser heredado. Sin embargo, nosotros guardamos también el luto por Derrida, éste se nos encomienda como tarea, como ceniza, y por tanto somos igualmente herederos de la relación entre ambos. Así, leer a Heidegger y a Derrida es asumir el im-posile ejercicio de darles lugar, de traducir interminablemente sus textos entre sí, prometiendo una reconciliación que no puede nunca llegar. Porque las resistencias entre ambos son muchas, a veces tan sutiles como una simple cuestión de tono ${ }^{109}$. Sin embargo, como se ha señalado anteriormente desde Derrida, es precisamente la imposibilidad de la traducción la que debe lanzarnos hacia ella, hacia la lectura interminable de estos textos, hacia el fracaso de su traducción que no es sino el mayor éxito, la posibilidad de lo im-posible, la promesa de la traducción.

Universidad Nacional de Educación a Distancia (España)

Pablo Bernardo Sánchez Gómez

pabersg@gmail.com

[Artículo aprobado para publicación en enero de 2019]

103 Derrida, J., «La mythologie blanche» en Marges de la philosophie, ed. cit., p. 255 (traducción española, p. 254).

104 Cfr. Derrida, J., «Le retrait de la métaphore» en Psyché. Inventions de l'autre, ed. cit., p. 82 .

105 DerridA, J., «La déconstruction et l'autre», ed. cit., p. 25.

106 DERrida, J., op. cit., p. 11.

107 Derrida, J., Positions, ed. cit., p. 18 (traducción española, p. 16).

108 Cfr. Janicaud, D., Heidegger en France II. Entretiens, Hachette, Paris, 2005, p. 103.

109 Cfr. Janicaud, D., op. cit., p. 116. 